OPEN ACCESS

Edited by: Brendan Bulfin,

ETH Zürich, Switzerland

Reviewed by:

Qiang Liu,

China University of Petroleum, China

Fubin Yang,

Beijing University of Technology,

China

Shiqi Huang,

Swiss Federal Institute of Technology

Lausanne, Switzerland

*Correspondence:

Chengzhen Sun

sun-cz@xitu.edu.cn

Specialty section:

This article was submitted to

Process and Energy Systems

Engineering,

a section of the journal

Frontiers in Energy Research

Received: 10 September 2021

Accepted: 29 October 2021

Published: 22 November 2021

Citation:

Sun C, Liu C, Luo K and Bai B (2021) Quasi-Unidirectional Transport Bilayer Two-Dimensional Nanopores for Highly-Efficient Molecular Sieving.

Front. Energy Res. 9:773924. doi: 10.3389/fenrg.2021.773924

\section{Quasi-Unidirectional Transport Bilayer Two-Dimensional Nanopores for Highly-Efficient Molecular Sieving}

\author{
Chengzhen Sun*, Cheng Liu, Kailin Luo and Bofeng Bai \\ State Key Laboratory of Multiphase Flow in Power Engineering, Xi'an Jiaotong University, X'an, China
}

Two-dimensional nanopores are very promising for high-permeance molecular sieving, but the molecular backflow from permeate-side to feed-side is not beneficial for improving molecular permeance. We study the quasi-unidirectional molecular transport through a graphene-hexagonal boron nitride bilayer nanopore, aiming to realize a high-permeance molecular sieving. Molecular dynamics simulations of $\mathrm{CO}_{2} / \mathrm{CH}_{4}$ separations show that the bilayer pore presents 3.7 times higher selectivity comparing to the single-layer graphene nanopore with the same size. The quasi-unidirectional molecular transport is attributed to the distinctive adsorption abilities of gas molecules on the two sides of bilayer nanopores and the inhibited molecular backflow from permeate-side to feed-side. This work provides a promising way to realize the ultra-permeable porous membranes with molecular permeance even higher than the single-layer atomic-thickness membranes.

Keywords: nanopore, molecular sieve, molecular dynamics, gas separation membrane, nanoporous graphene

\section{INTRODUCTION}

Graphene (Geim and Novoselov, 2007; Geim, 2009) and its derivatives have been proposed as a perfect candidate for the molecular separation membranes owing to its atomic thickness, because the permeance of membrane is generally inversely proportional to the thickness of membrane materials. Thus, the one-atomic thickness graphene can be a high-efficiency separation membrane with a high molecular permeance (Wen et al., 2015; Sun et al., 2020a). To realize the graphene-based membranes, the selective nanopores should be introduced into the impermeable pristine graphene to generate the nanoporous graphene (NPG) sheets (Koenig et al., 2012). In 2009, Jiang et al. (Jiang et al., 2009) firstly demonstrated that the NPGs can realize the high-efficiency separation of $\mathrm{H}_{2} / \mathrm{CH}_{4}$ mixtures with a high $\mathrm{H}_{2}$ permeance of $2.985 \times 10^{9} \mathrm{GPU}\left(1 \mathrm{GPU}=3.35 \times 10^{-10} \mathrm{~mol} / \mathrm{s} \mathrm{m}^{2} \mathrm{~Pa}\right)$. Currently, the industrial-scale NPG membranes have been successfully synthesized with a high molecular permeance as the theoretically expected (Boutilier et al., 2017; Wang et al., 2017; Zhao et al., 2019). Meanwhile, the molecular permeation mechanisms through NPG membranes were also well revealed (Drahushuk and Strano, 2012; Sun et al., 2014; Yuan et al., 2017; Sun et al., 2019a; Sun et al., 2021a; Sun et al., 2021b). In short, the NPG membranes for molecular separation has become a reality, especially for the application of gas separation.

For the NPG membranes, the structure of selective nanopores in graphene is strictly symmetrical, accordingly molecules can permeate through the graphene nanopores from both sides in two directions. Unlike other one-way permeable membranes, the molecular permeation through NPG membranes can appear the backflow from permeate-side to feed-side, which would greatly decrease the net permeation rate of NPG membranes. If the backflow can be effectively inhibited or totally eliminated, the permeance of NPG membranes would be further improved. 


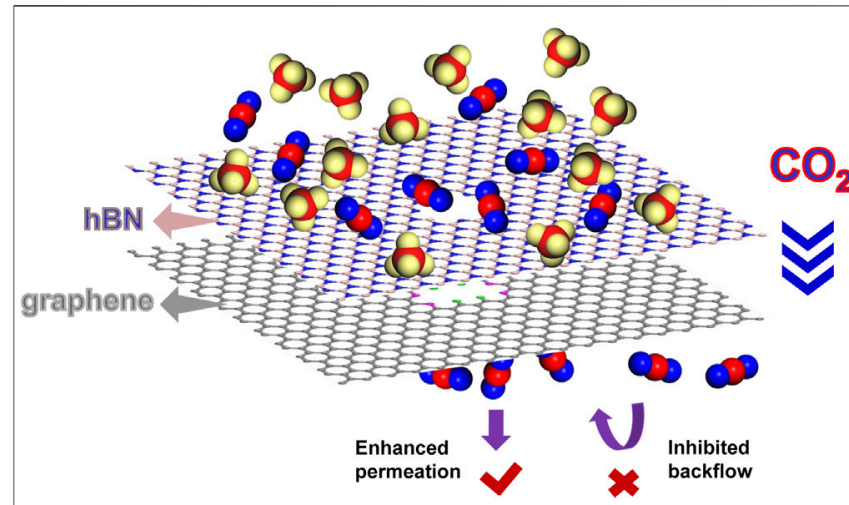

GRAPHICAL ABSTRACT

Hexagonal boron nitride ( $\mathrm{hBN})$, another type of twodimensional material, has become a hot topic of research thanks to its structural equivalency to graphene and its outstanding mechanical (Chen et al., 2015), thermodynamic (Liu and Turner, 2014), and electronic properties (Li et al., 2012). hBN is composed of the heterogeneous boron and nitrogen atoms and thus it would exhibit a stronger molecular adsorption ability comparing to the graphene. Therefore, we adopt $\mathrm{hBN}$ and graphene hybrid sheets to form a two-dimensional membrane with stronger adsorption surface in one side and weaker adsorption surface in another side. In this study, we systematically study the quasi-unidirectional molecular transport characteristics through a graphene-hBN bilayer nanopore, following the asymmetric two-layer porous membranes proposed by Liu et al. (Liu et al., 2020). We show that the quasi-unidirectional molecular transport is realized by inhibiting the molecular backflow from permeate-side to feed-side. Through molecular dynamics (MD) simulations of the $\mathrm{CO}_{2} / \mathrm{CH}_{4}$ separation, it is shown that the permeance of the graphene-hBN bilayer nanopore is higher than that of the monolayer graphene nanopore with the same size. Even more, the selectivity of molecular separation is improved by employing the bilayer twodimensional nanopores. This enhancement of the molecular permeance is caused by the relatively stronger $\mathrm{CO}_{2}$ adsorption abilities on the hBN surfaces compared with those on the graphene surface. It is expected that this type of graphene nanopores are promising for high-efficiency membranes for molecular separation as well as other processes involving molecular permeation (Sun et al., 2020b). This work presents a promising road to achieve ultrapermeable porous membranes even with a higher molecular permeability compared to the single-layer membranes. Furthermore, the stacking of multiple atomic layers makes such membranes more feasible for large-area industrial production.

\section{SIMULATION MODEL}

\subsection{Pore Structure}

During the molecular permeation, the hBN surface is towards the feed-side while the graphene surface is towards the permeateside. Thus, the $\mathrm{CO}_{2} / \mathrm{CH}_{4}$ mixtures are initially arranged in the
$\mathrm{hBN}$ side. The graphene-hBN bilayer nanopore is constructed by stacking the graphene and $\mathrm{hBN}$ layers together and generating a nanopore with the same size (Figure 1A). To realize a selectivity of the separation of $\mathrm{CO}_{2} / \mathrm{CH}_{4}$ mixtures, the pores are functionalized by the $\mathrm{N}$ and $\mathrm{H}$ atoms (Figure 1B), as done in our early work (Sun and Bai, 2017). The N functionalization not only enlarges the size of the pore to achieve a high permeance of $\mathrm{CO}_{2}$ molecules, but also enhances the adsorption intensities of $\mathrm{CO}_{2}$ molecules on the surface which further improves the permeability and selectivity. The spacing distance of two layers is set as $3.4 \AA$ in the simulation (Figure 1C).

\subsection{Simulation System and Method}

To examine the sieving effect of nanopores, we perform MD simulations for the separation of $\mathrm{CO}_{2} / \mathrm{CH}_{4}$ mixtures in a nonequilibrium system based on the platform of LAMMPS. In the system, there are 1,000 mixture gas molecules, namely $500 \mathrm{CO}_{2}$ molecules and $500 \mathrm{CH}_{4}$ molecules. Initially, the molecules are arranged alternatively and uniformly in the feed side, while the permeate side is kept vacuum, as seen from Figure 1A. The nanopore is located at the center of the simulation box of height $140 \mathrm{~nm}$. The heights of box in the permeate side and feed side are both $66.6 \mathrm{~nm}$. Periodic boundary conditions are applied in the $x$ and $y$-directions (parallel to the surface), while reflective wall condition is applied in the $z$-direction (perpendicular to the surface). The spacing distance among the nanopores in the porous membranes is assigned as $4 \mathrm{~nm}$. In this case, the number density of the pores is $6.25 \times 10^{12} \mathrm{~cm}^{-2}$, which is comparable with the pore densities in the fabricated NPG membranes in laboratory (O'Hern et al., 2014). Owing to the application of periodic boundary conditions, the area of sheet is assigned as $4 \times 4 \mathrm{~nm}^{2}$ based on the spacing distance among nanopores. The simulation is run in a NVT ensemble with a temperature of $350 \mathrm{~K}$, which is close to the running temperature in gas separation industry. A simulation period of $6.6 \times 10^{7}$ timesteps (time step is $0.3 \mathrm{fs}$ ) is chosen such that the permeable gases transport through the nanopore with a relatively fast rate, ensuring a high accuracy in the calculation of permeance. Based on the ideal gas equation, the initial pressure of the feed side is estimated as 21.1 bar.

For carbon and hydrogen atoms in graphene sheet and $\mathrm{CH}_{4}$ molecules, the atomic interactions are modeled by the AIREBO potential model; while for $\mathrm{CO}_{2}$ molecules and hBN sheets, they are modeled by the Lennard-Jones (LJ) potential model. The detailed introduction and parameters of AIREBO potential can be found in the literature (Stuart et al., 2000). A three-site model with three partial charges is adopted for $\mathrm{CO}_{2}$ molecules. In the model, the LJ potentials and Coulombic potentials are coupled together, as follows:

$$
\phi\left(r_{i j}\right)=\left\{\begin{array}{c}
4 \varepsilon\left[\left(\frac{\sigma}{r_{i j}}\right)^{12}-\left(\frac{\sigma}{r_{i j}}\right)^{6}\right]+\frac{C q_{i} q_{j}}{\chi r_{i j}}\left(r_{i j}<r_{c u t}\right) \\
0 \quad\left(r_{i j} \geq r_{c u t}\right)
\end{array}\right.
$$

where $\sigma$ is length scale, $\varepsilon$ is energy scale, $q_{\mathrm{i}}$ and $q_{\mathrm{j}}$ are the charges on atom $\mathrm{i}$ and atom $\mathrm{j}, C$ is an energy-conversion constant, and $\chi$ is 

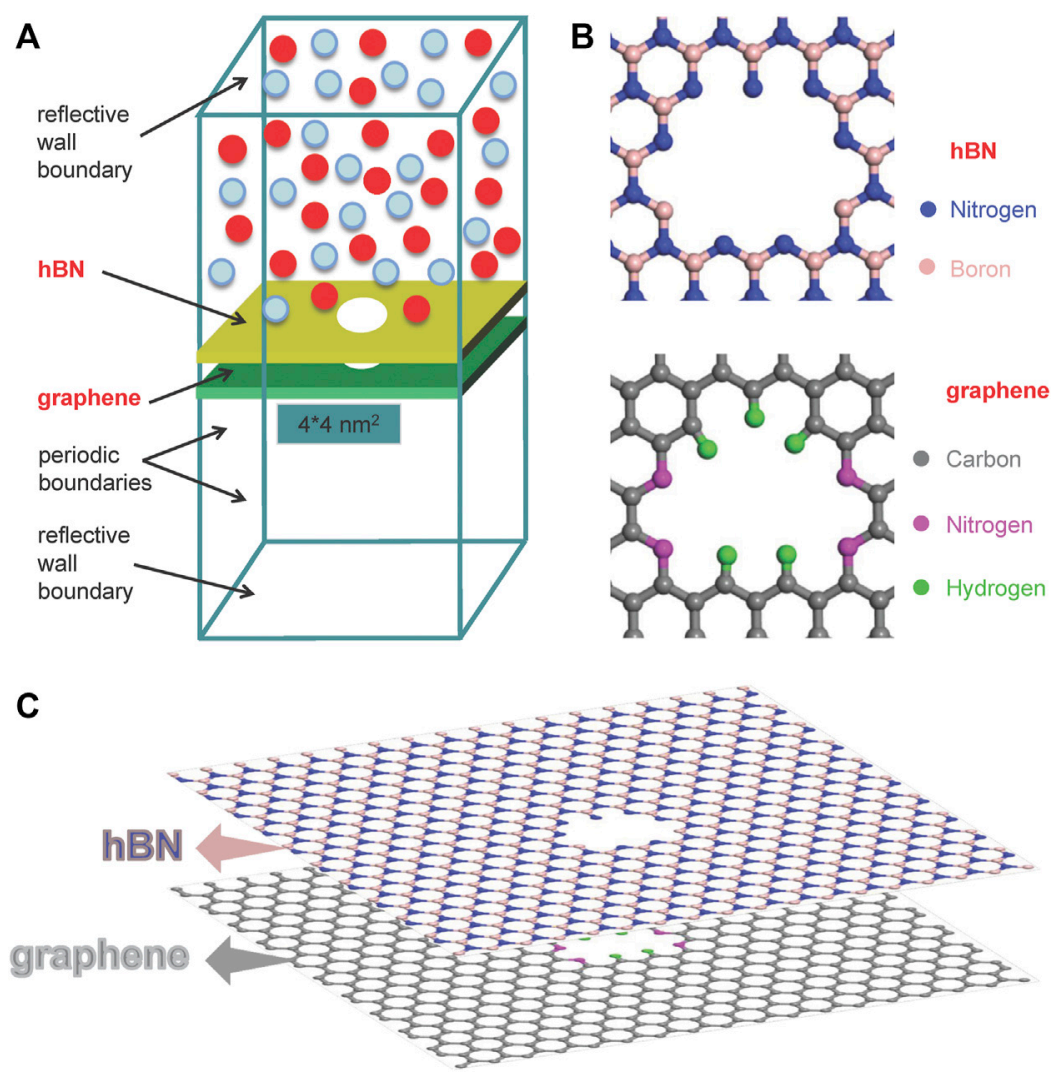

FIGURE 1 | Simulation model. (A) Non-equilibrium MD simulation system; (B) structure of the nanopore; (C) stacking of the graphene and hBN layers.

the dielectric constant. The parameters for cross atoms are estimated by the Lorentz-Berthelot mixing rules based on the following parameters for C-C interactions: $\sigma=3.40 \AA, \varepsilon=2.413 \times$ $10^{-3} \mathrm{eV}$ (Du et al., 2011), H-H interactions: $\sigma=2.50 \AA, \varepsilon=1.300 \times$ $10^{-3} \mathrm{eV}$ (Liu et al., 2015), and $\mathrm{N}_{\text {graphene }} \mathrm{N}_{\text {graphene }}$ interactions: $\sigma=$ $3.297 \AA, \varepsilon=3.126 \times 10^{-3} \mathrm{eV}$ (Chae and Violi, 2011), B-B interactions: $\sigma=3.453 \AA, \varepsilon=4.116 \times 10^{-3} \mathrm{eV}$ (Baowan and Hill, 2007), $\mathrm{N}_{\mathrm{hBN}}-\mathrm{N}_{\mathrm{hBN}}$ interactions: $\sigma=3.365 \AA, \varepsilon=6.281 \times$ $10^{-3} \mathrm{eV}$ (Baowan and Hill, 2007). The bond and angle potentials in the gas molecules $\left(\mathrm{CO}_{2}\right)$ and the functionalized groups are all modeled by harmonic potential. The parameters in these potential models can refer to our previous work (Sun and Bai, 2017). The charges on the atoms near the pore are obtained through a density functional theory calculation using the $\mathrm{DMol}_{3}$ module in Material Studio software.

\section{RESULTS AND DISCUSSION}

\subsection{Molecular Permeance}

As seen from Figure 2A, the number of permeated molecules increases over time but the increasing rate slows down gradually owing to the non-equilibrium permeation process. The molecular number in the permeate side $(z<-0.6 \mathrm{~nm})$ of permeating molecules sharply increases with the passage of time, while that of non-permeating molecules increases very slowly and only a few $\mathrm{CH}_{4}$ molecules appear in the permeate-side. Namely, the graphene-hBN bilayer nanopore exhibits a phenomenon of selective molecular sieving for the separation of $\mathrm{CO}_{2} / \mathrm{CH}_{4}$ mixtures. Comparing to the single-layer graphene nanopore, the performance of selective molecular sieving of graphene-hBN bilayer nanopore is more significant.

In order to quantitatively analyze the molecular sieving performance of nanopores, the molecular permeance is calculated based on the relationship between the number of permeated molecules $N$ and time $t$, as follows (Sun and Bai, 2017):

$$
N=\left(250-N_{a l} / 2\right) \times\left(1-e^{-8.24 \times 10^{10} P t}\right)
$$

where $N_{\mathrm{al}}$ is the average number of molecules adsorbed on both sides of graphene, $P$ is molecular permeance, and the constant 250 is related to the molecular number 500 in the simulation box. On the basis of this function, the curves of molecular number $N$ versus time $t$ are fitted and the molecular permeance $P$ is then obtained.

The molecular permeance $(P)$ and selectivity for the separation of $\mathrm{CO}_{2} / \mathrm{CH}_{4}$ mixtures through the bilayer and single-layer nanopores are displayed in Figure 2B. For $\mathrm{CO}_{2} / \mathrm{CH}_{4}$, the $\mathrm{CO}_{2}$ permeance of bilayer nanopore is higher than that of single-layer nanopore while the $\mathrm{CH}_{4}$ permeance is lower, accordingly a higher selectivity appears for the bilayer nanopore. The bilayer pore 


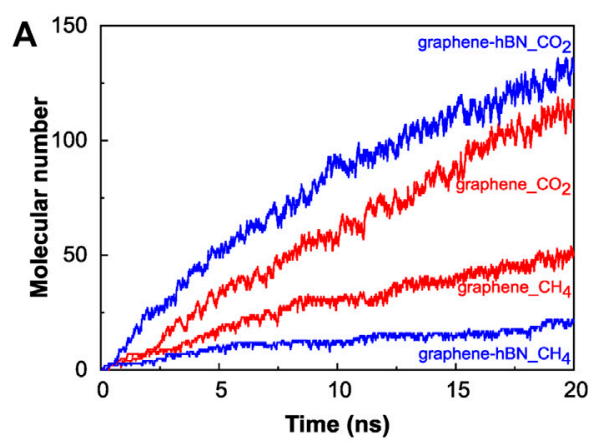

B

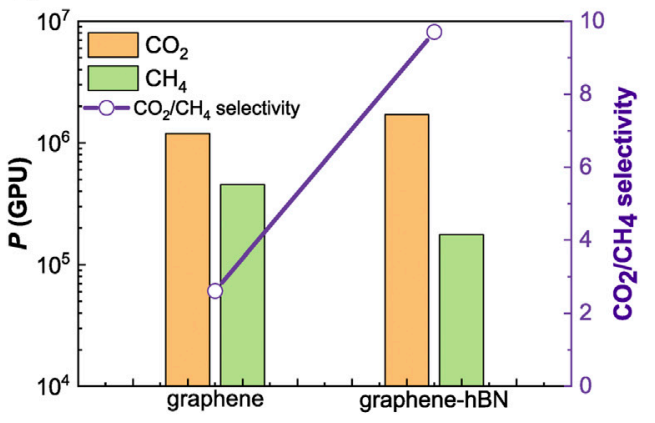

C

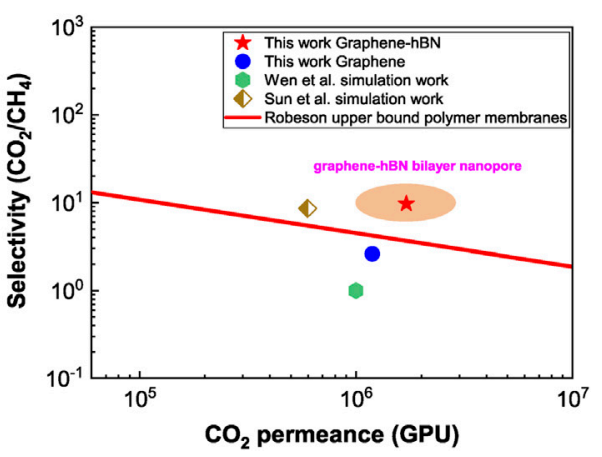

FIGURE 2 | $\mathrm{CO}_{2} / \mathrm{CH}_{4}$ separation performance of nanopores. (A) Time-variation of the number of permeated molecules for the separation of $\mathrm{CO}_{2} / \mathrm{CH}_{4}$; (B) comparison of the molecular permeance and selectivity; (C) comparison with existing simulation data in literature (Wen et al., 2015; Sun and Bai, 2017).

presents a 3.7 times higher selectivity comparing to the singlelayer graphene nanopore with the same size. Meanwhile, we compare the separation performance of the bilayer nanopore and graphene nanopore with available simulation data of NPG membranes in literature (Wen et al., 2015; Sun and Bai, 2017), as shown in Figure 2C. It can be found that both the single-layer graphene and bilayer nanopores show a high molecular permeance. The graphene-hBN bilayer nanopore exhibits an ultra-high permeance and high selectivity, such that its separation performance exceeds the upper bound of polymer membranes.

\subsection{Molecular Adsorption}

To understand why the graphene-hBN bilayer nanopore can present the quasi-unidirectional transport characteristic and finally exhibit a better molecular separation performance comparing to the single-layer nanopore, we firstly analyze the molecular adsorption characteristics on the two sides of the graphene-hBN bilayer surface and single-layer graphene surface. From the number density distributions of gas molecules along the perpendicular direction (Figure 3A,B), it can be found that the density of adsorbed molecules for $\mathrm{CO}_{2}$ is higher than that of $\mathrm{CH}_{4}$. This means that the interactions between $\mathrm{CO}_{2}$ molecules and hBN or graphene surfaces are stronger than those of $\mathrm{CH}_{4}$ molecules. Owing to the non-equilibrium permeation process, the density of adsorbed molecules in permeate side is weaker than that in feed side. Comparisons between the bilayer surface and single-layer surface in Figure 3C show that the bilayer nanopore has a stronger adsorption ability of $\mathrm{CO}_{2}$ gas molecules because the interaction of the hBN surface is greater than that of graphene surface. Meanwhile, the competitive adsorption of $\mathrm{CO}_{2}$ and $\mathrm{CH}_{4}$ results in a reduction in the adsorption density of $\mathrm{CH}_{4}$ on the feed-side surface of bilayer nanopore.

The distinctive molecular adsorption on the surface can partially explain why the graphene-hBN bilayer nanopore can improve selectivity while maintain ultra-high permeability. The adsorption of gas molecules on the surfaces is beneficial for the molecular permeation through the nanopore (Drahushuk and Strano, 2012; Sun et al., 2014), therefore the stronger molecular adsorption in the feed side can promote the molecular permeation from feed-side to permeate-side. Thus, the distinctive adsorption abilities of $\mathrm{CO}_{2}$ and $\mathrm{CH}_{4}$ molecules on the feed side of the graphene-hBN bilayer surface is the main reason for the high-efficiency molecular sieving.

The molecular adsorption on the graphene surface may have another positive contribution on the molecular permeance by inhibiting molecular backflow from permeate-side to feed-side. This inhibition effect can be well analyzed from the aspect of molecular trajectories during permeation. We obtain the molecular crossing number $N_{\mathrm{F}-\mathrm{P}}$ and $N_{\mathrm{P}-\mathrm{F}}$, respectively; $N_{\mathrm{F}-\mathrm{P}}$ is the molecular crossing number from feed-side to permeate-side, while $N_{\mathrm{P}-\mathrm{F}}$ is the molecular crossing number from permeate-side to feed-side. Figure $4 \mathbf{A}$ 

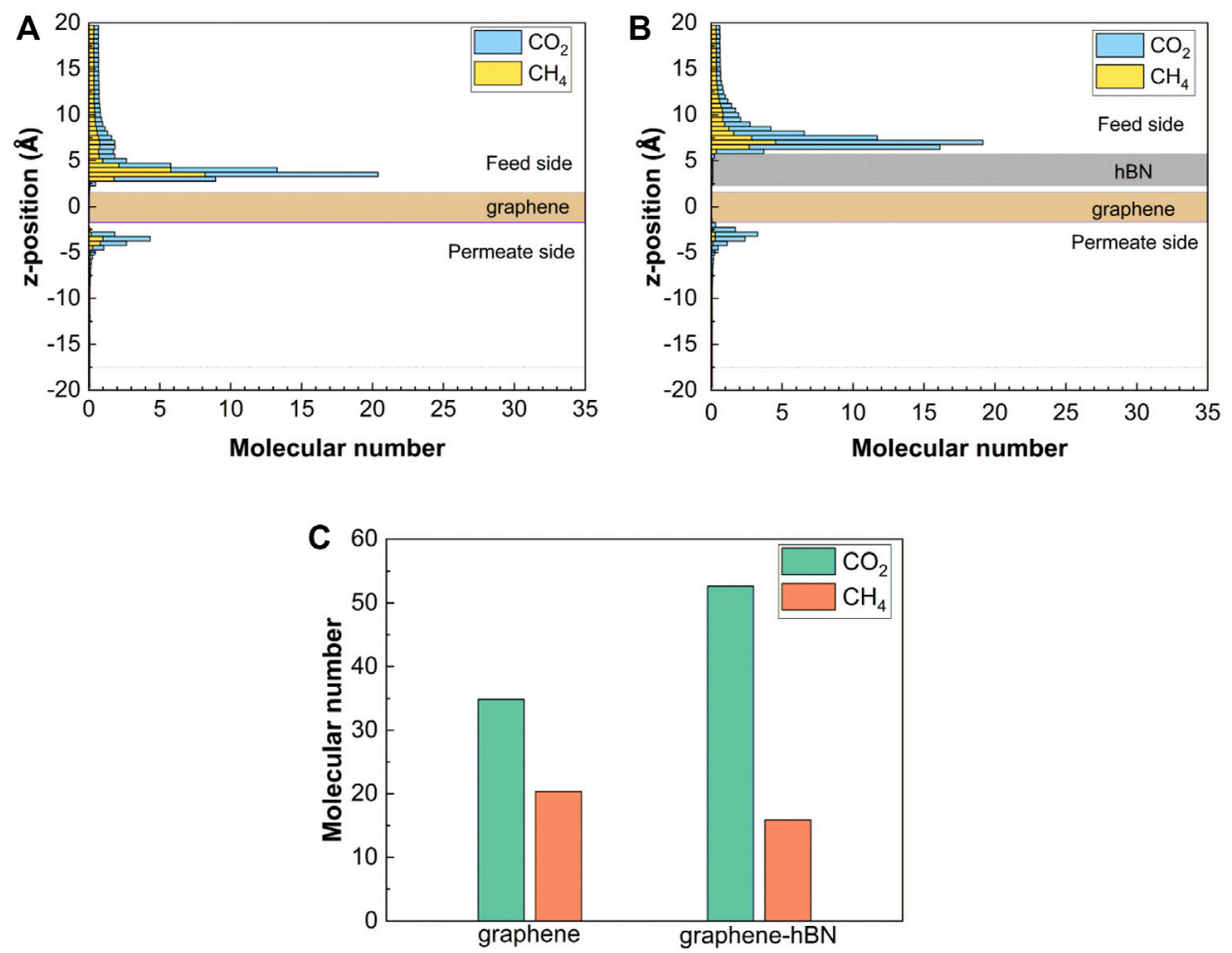

FIGURE 3|Molecular adsorption characteristics on the two sides of surface. (A) Number density distributions of the molecules along the perpendicular direction on the graphene surface; (B) number density distributions of the molecules along the perpendicular direction on the graphene-hBN surface; (C) number of adsorbed molecules on the feed-side surface.

shows the time variations of $N_{\mathrm{F}-\mathrm{P}}, N_{\mathrm{P}-\mathrm{F}}$ for the $\mathrm{CO}_{2}$ molecules. Over time, the two numbers all necessarily increase gradually with the permeation of molecules, but $N_{\mathrm{F}-\mathrm{P}}$ increases more sharply and $N_{\mathrm{P}-\mathrm{F}}$ increases very slowly. Interestingly, for the bilayer nanopore $N_{\mathrm{F}-\mathrm{P}}$ increases while $N_{\mathrm{P}-\mathrm{F}}$ decreases comparing to the single-layer nanopore, demonstrating that the bilayer nanopore can promote the molecular permeation from feed-side to permeate-side while inhibit the molecular backflow. This phenomenon exactly proves the quasiunidirectional transport characteristics of the graphene-hBN bilayer nanopore.

Then, we further obtain the interacting energy distributions between gas molecules and graphene-hBN bilayer nanopore by calculating the potential of mean force (PMF). The PMF profiles of gas molecules passing through the nanopore are calculated based on the sampling method of Adaptive Biasing Force (Sun
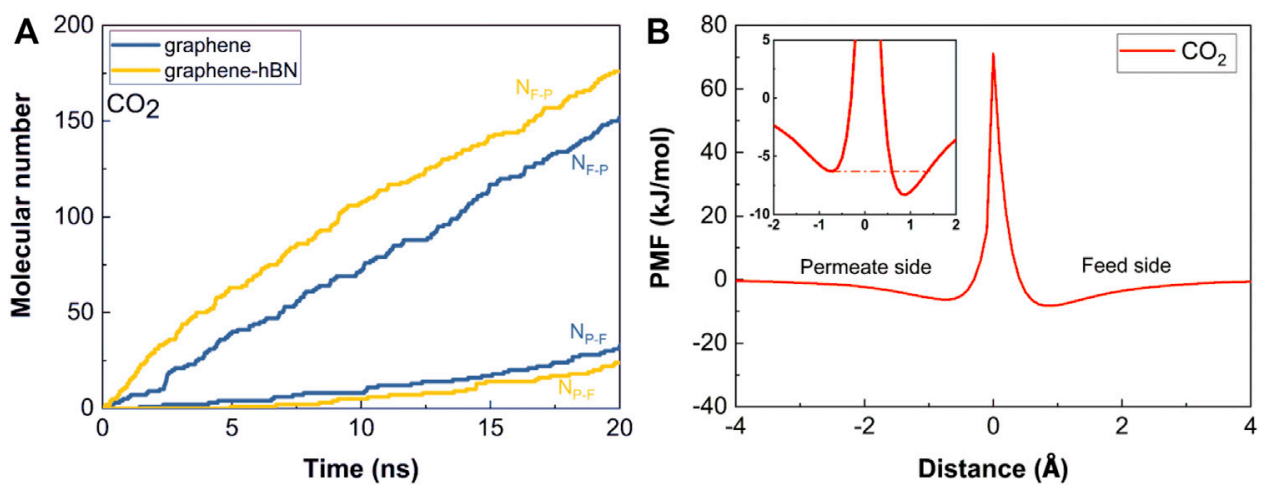

FIGURE 4 | Quasi-unidirectional gas transport. (A) Time variations of the number of molecular crossings in two directions for the separation of $\mathrm{CO}_{2} / \mathrm{CH}_{4}$; (B) $\mathrm{PMF}$ profiles of $\mathrm{CO}_{2}$ molecules passing through the bilayer nanopore. 
et al., 2019b). It can be clearly seen from Figure 4B that the PMF distribution is asymmetric, namely, $\mathrm{CO}_{2}$ molecules have a relatively deeper attractive potential well in the feed side comparing to the permeate side. This further proves that there is a stronger interaction between gas molecules and graphene$\mathrm{hBN}$ bilayer surface in feed side. PMF calculations give a strong evidence for the distinctive molecular adsorption abilities on the two sides and the resulted quasi-unidirectional molecular transport characteristics from the insight of thermodynamic physics.

\section{CONCLUSION}

We study the quasi-unidirectional transport characteristics through a graphene-hBN bilayer nanopore to realize highefficient molecular sieving. Compared to the single-layer graphene nanopores, the bilayer nanopores exhibit both higher molecular permeance and selectivity for the separation of $\mathrm{CO}_{2}$ / $\mathrm{CH}_{4}$ mixtures. For the bilayer nanopore, the molecular permeation from feed-side to permeate-side is enhanced while the molecular crossings from permeate-side to feed-side (molecular backflow) are weakened. The quasi-unidirectional transport characteristics are caused by the distinctive molecular adsorption abilities on two sides of the graphenehBN bilayer surface, which are confirmed from the molecular number density distributions perpendicular to the surface and PMF profiles of gas molecules passing through the nanopore. This work provides a promising way to realize the ultra-

\section{REFERENCES}

Baowan, D., and Hill, J. M. (2007). Nested boron Nitride and Carbon-boron Nitride Nanocones. Micro Nano Lett. 2, 46-49. doi:10.1049/mnl:20070041

Boutilier, M. S. H., Jang, D., Idrobo, J.-C., Kidambi, P. R., Hadjiconstantinou, N. G., and Karnik, R. (2017). Molecular Sieving across Centimeter-Scale Single-Layer Nanoporous Graphene Membranes. Acs Nano. 11, 5726-5736. doi:10.1021/ acsnano.7b01231

Chae, K., and Violi, A. (2011). Mutual Diffusion Coefficients of Heptane Isomers in Nitrogen: A Molecular Dynamics Study. J. Chem. Phys. 134, 044537. doi:10.1063/1.3512918

Chen, X., Zhang, L., Park, C., Fay, C. C., Wang, X., and Ke, C. (2015). Mechanical Strength of boron Nitride Nanotube-Polymer Interfaces. Appl. Phys. Lett. 107, 253105. doi:10.1063/1.4936755

Drahushuk, L. W., and Strano, M. S. (2012). Mechanisms of Gas Permeation through Single Layer Graphene Membranes. Langmuir. 28, 16671-16678. doi:10.1021/la303468r

Du, H., Li, J., Zhang, J., Su, G., Li, X., and Zhao, Y. (2011). Separation of Hydrogen and Nitrogen Gases with Porous Graphene Membrane. J. Phys. Chem. C 115, 23261-23266. doi:10.1021/jp206258u

Geim, A. K. (2009). Graphene: Status and Prospects. Science 324, 1530-1534. doi:10.1126/science.1158877

Geim, A. K., and Novoselov, K. S. (2007). The Rise of Graphene. Nat. Mater 6, 183-191. doi:10.1038/nmat1849

Jiang, D.-e., Cooper, V. R., and Dai, S. (2009). Porous Graphene as the Ultimate Membrane for Gas Separation. Nano Lett. 9, 4019-4024. doi:10.1021/nl9021946

Koenig, S. P., Wang, L., Pellegrino, J., and Bunch, J. S. (2012). Selective Molecular Sieving through Porous Graphene. Nat. Nanotech 7, 728-732. doi:10.1038/ nnano.2012.162 permeable porous membranes with molecular permeance even higher than the single-layer graphene membranes. Meanwhile, the fabrication of such two-layer membranes is instead more feasible comparing to the single-layer graphene membranes because of the elimination of intrinsic defects via the overlap of atomic-layer sheets.

\section{DATA AVAILABILITY STATEMENT}

The original contributions presented in the study are included in the article, further inquiries can be directed to the corresponding author.

\section{AUTHOR CONTRIBUTIONS}

CS performed the simulations, analyzed the data, wrote the first draft of manuscript and revised the manuscript. CL and KL analyzed the data and discussed the simulation results. BB conceived and designed the simulations. All authors contributed to manuscript revision, read, and approved the submitted version.

\section{FUNDING}

National Natural Science Foundation of China for general project No. 51876169.

Li, X., Wu, X., Zeng, X. C., and Yang, J. (2012). Band-gap Engineering via Tailored Line Defects in boron-nitride Nanoribbons, Sheets, and Nanotubes. Acs Nano 6, 4104-4112. doi:10.1021/nn300495t

Liu, H., Chen, Z., Dai, S., and Jiang, D.-e. (2015). Selectivity Trend of Gas Separation through Nanoporous Graphene. J. Solid State. Chem. 224, 2-6. doi:10.1016/j.jssc.2014.01.030

Liu, H., and Turner, C. H. (2014). Adsorption Properties of Nitrogen Dioxide on Hybrid Carbon and boron-nitride Nanotubes. Phys. Chem. Chem. Phys. 16, 22853-22860. doi:10.1039/c4cp03555k

Liu, M., Song, D., Wang, X., Sun, C., and Jing, D. (2020). Asymmetric Two-Layer Porous Membrane for Gas Separation. J. Phys. Chem. Lett. 11, 6359-6363. doi:10.1021/acs.jpclett.0c01797

O’Hern, S. C., Boutilier, M. S., Idrobo, J. C., Song, Y., Kong, J., Laoui, T., et al. (2014). Selective Ionic Transport through Tunable Subnanometer Pores in Single-Layer Graphene Membranes. Nano Lett. 14, 1234-1241. doi:10.1021/nl404118f

Stuart, S. J., Tutein, A. B., and Harrison, J. A. (2000). A Reactive Potential for Hydrocarbons with Intermolecular Interactions. J. Chem. Phys. 112, 6472-6486. doi: $10.1063 / 1.481208$

Sun, C., and Bai, B. (2017). Molecular Sieving through a Graphene Nanopore: Nonequilibrium Molecular Dynamics Simulation. Sci. Bull. 62, 554-562. doi:10.1016/j.scib.2017.03.004

Sun, C., Boutilier, M. S. H., Au, H., Poesio, P., Bai, B., Karnik, R., et al. (2014). Mechanisms of Molecular Permeation through Nanoporous Graphene Membranes. Langmuir. 30, 675-682. doi:10.1021/la403969g

Sun, C., Liu, M., and Bai, B. (2019a). Molecular Simulations on Graphene-Based Membranes. Carbon. 153, 481-494. doi:10.1016/j.carbon.2019.07.052

Sun, C., Luo, K., Zhou, R., and Bai, B. (2021a). Theoretical Description of Molecular Permeation via Surface Diffusion through Graphene Nanopores. Phys. Chem. Chem. Phys. 23, 7057-7065. doi:10.1039/d0cp05629d

Sun, C., Zhou, R., and Bai, B. (2020a). Electrostatic Effect-Based Selective Permeation Characteristics of Graphene Nanopores. Acta Phys. -Chim. Sin. 36, 1911044. 
Sun, C., Zhou, R., Bai, B., Lin, Y., and Li, B. (2021b). Multilayer Graphene Sheet with Conical Nanopores as a Membrane for High-Permeance Molecular Separation. J. Phys. Chem. C. 125, 3047-3054. doi:10.1021/ acs.jpcc.0c10717

Sun, C., Zhou, R., Zhao, Z., and Bai, B. (2020b). Nanoconfined Fluids: What Can We Expect from Them? J. Phys. Chem. Lett. 11, 4678-4692. doi:10.1021/ acs.jpclett.0c00591

Sun, C., Zhu, S., Liu, M., Shen, S., and Bai, B. (2019b). Selective Molecular Sieving through a Large Graphene Nanopore with Surface Charges. J. Phys. Chem. Lett. 10, 7188-7194. doi:10.1021/acs.jpclett.9b02715

Wang, L., Boutilier, M. S. H., Kidambi, P. R., Jang, D., Hadjiconstantinou, N. G., and Karnik, R. (2017). Fundamental Transport Mechanisms, Fabrication and Potential Applications of Nanoporous Atomically Thin Membranes. Nat. Nanotech. 12, 509-522. doi:10.1038/nnano.2017.72

Wen, B. Y., Sun, C. Z., and Bai, B. F. (2015). Molecular Dynamics Simulation of the Separation of $\mathrm{CH} 4 / \mathrm{CO} 2$ by Nanoporous Graphene. Acta Physico-Chimica Sinica. 31, 261-267.

Yuan, Z., Govind Rajan, A., Misra, R. P., Drahushuk, L. W., Agrawal, K. V., Strano, M. S., et al. (2017). Mechanism and Prediction of Gas Permeation through Subnanometer Graphene Pores: Comparison of Theory and Simulation. Acs Nano. 11, 7974-7987. doi:10.1021/acsnano.7b02523
Zhao, J., He, G., Huang, S., Villalobos, L. F., Dakhchoune, M., Bassas, H., et al. (2019). Etching Gas-Sieving Nanopores in Single-Layer Graphene with an Angstrom Precision for High-Performance Gas Mixture Separation. Sci. Adv. 5, eaav1851. doi:10.1126/sciadv.aav1851

Conflict of Interest: The authors declare that the research was conducted in the absence of any commercial or financial relationships that could be construed as a potential conflict of interest.

Publisher's Note: All claims expressed in this article are solely those of the authors and do not necessarily represent those of their affiliated organizations, or those of the publisher, the editors and the reviewers. Any product that may be evaluated in this article, or claim that may be made by its manufacturer, is not guaranteed or endorsed by the publisher.

Copyright (C) 2021 Sun, Liu, Luo and Bai. This is an open-access article distributed under the terms of the Creative Commons Attribution License (CC BY). The use, distribution or reproduction in other forums is permitted, provided the original author(s) and the copyright owner(s) are credited and that the original publication in this journal is cited, in accordance with accepted academic practice. No use, distribution or reproduction is permitted which does not comply with these terms. 